Revue d'histoire de l'Amérique française

ZRS REVUE D.HISTOIRE DE L'AMÉRIQUE FRANÇAISE

\title{
Gens de couleur libres et colons de Saint-Domingue devant la Constituante (3e partie et fin)
}

\section{Gabriel Debien}

Volume 4, numéro 4, mars 1951

URI : https://id.erudit.org/iderudit/801669ar

DOI : https://doi.org/10.7202/801669ar

Aller au sommaire du numéro

Éditeur(s)

Institut d'histoire de l'Amérique française

ISSN

0035-2357 (imprimé)

1492-1383 (numérique)

Découvrir la revue

Citer cet article

Debien, G. (1951). Gens de couleur libres et colons de Saint-Domingue devant la Constituante (3e partie et fin). Revue d'histoire de l'Amérique française, 4(4),

530-549. https://doi.org/10.7202/801669ar d'utilisation que vous pouvez consulter en ligne. 


\section{GENS DE COULEUR LIBRES ET COLONS DE SAINT-DOMINGUE DEVANT LA CONSTITUANTE}

(马e partie et fin)*

IX

\section{DEVANT L'ASSEMBLÉE DES REPRÉSENTANTS DE LA VILLE DE PARIS}

\section{(1er-11 février 1790)}

Dejoly ni les gens de couleur n'avaient renoncé a faire aboutir leurs demandes. Ils avaient maintenant un défenseur à la Constituante dans la personne de l'abbé Grégoire. Il s'agissait d'éclairer les membres du Comité de vérification et l'opinion. Ce fut le but du Mémoire en faveur des gens de couleur que Grégoire publia dans le courant de décembre ${ }^{124}$. Il reprenait la description de l'état des libres qu'il avait esquissée le 3 décembre devant la Constituante et les arguments qui démontraient la justice, les avantages même de leur émancipation et de leur admission à l'exercice de tous les droits politiques. Mais réfute-t-on jamais des préjugés! Il concluait par proposer un décret qui déclarerait citoyens tous les libres et admettrait provisoirement à l'Assemblée cinq députés à élire parmi les gens de couleur résidant à Paris.

Par malheur cette brochure paraissait au moment où des événements de Saint-Domingue tenaient aussi une grande place dans les conversations et dans les journaux. Des lettres du Cap, datées du

\footnotetext{
* Voir, pour première partie de cet article, Revue d'Histoire de l'Amérique française, III, no 2 (septembre 1950): 211-232; IV, no 3 (dcembre 1950): 398-426.

124. Adresse d l'Assemblee Nationale (Paris, Belin, 1789), 59 pages, in-8.
} 
mois de novembre, annonçaient des rassemblements de libres en plusieurs paroisses du Sud et même à Saint-Marc où un économe de plantation avait été tué par un atelier ${ }^{125}$ à cause de sa couleur ${ }^{126}$. Les sang-mêlés de Saint-Marc avaient délivré deux des leurs prisonniers pour dettes ${ }^{127}$. Tout cela avait excité beaucoup de colère chez les colons. Cependant la résistance n'était pas la même d'un bout a l'autre de la colonie: quelques paroisses du Sud avaient admis les libres aux assemblées primaires; aux Cayes, deux mulâtres avaient fait partie du Comité ${ }^{128}$. Mais ici et là, ce n'avait été qu'un feu de paille éteint par des protestations ou des échauffourées. Il fut interdit aux libres de s'assembler pour la rédaction de leurs cahiers et pour l'élection de leurs députés. En quelques paroisses on leur imposa même de reconnaître indirectement les députés blancs en les faisant souscrire aux subventions qu'on assurait aux représentants ${ }^{129}$. Au Petit-Goave, les libres avaient demandé leur admission à l'assemblée primaire et Ferrand de Baudière, ancien sénéchal, soupçonné d'avoir rédigé leur pétition, était mis à mort avec un mulâtre, Sanite d'Opsom, après un simulacre de jugement ${ }^{130}$. A Aquin, Guillaume Labadie, un des correspondants de Raimond, quarteron et propriétaire, est assailli chez lui, blessé d'un coup de feu, roué de coups et traîné trois lieues à la queue d'un cheval. Son fils est tué près de lui. A Jacmel, un grif est massacré. Tout se passait comme si on voulait faire peur aux gens de couleur qui agissaient en France.

De pareilles nouvelles faisaient crier contre Grégoire et sa brochure. Les colons avaient beau jeu de soutenir que le Mémoire en faveur des gens de couleur était sur le point "de mettre le feu aux

125. On appelait ainsi les esclaves travaillant sur la même plantation. $86 / 826$.

126. Arch. Nat., Dxxv 89/842 bis. Procès-verbal du 25 janvier 1790 et Dxxv

127. Garran Coulon, Rapport sur les troubles de Saint-Domingue (4 vol., Paris, ans V-VII), I: 107 et AN, W 15, Procès-verbal du 27 décembre 1789.

128. Ibid., Garran Coulon, I: 106 et Blanche Maurel, "Un député de SaintDomingue à la Constituante, J.-B. Gérard", Revue d'Histoire moderne (1934), 242; Debats sur l'affaire des colonies, I: 99 et 197.

129. Rd P. Cabon, Histoire d'Haïti (4 vol., s.d. Port-au-Prince), III: 32.

130. Précis historique des Annales de la colonie française (ms. Arch. Vienne, dépôt 102), I: 16. 
colonies"'131. Les faits répondaient à leurs alarmes. Ce n'était pas suffisant. A une brochure une brochure devait répondre. Moreau de Saint-Méry se chargea ou fut chargé de l'écrire. Ce sont les $O b$ servations d'un habitant des colonies sur le Mémoire en faveur des gens de couleur ou sang-mêlés de Saint-Domingue... adressé à l'Assemblée Nationale par $M$. Grégoire ${ }^{132}$ qui parurent anonymement, ce qui n'était pas pour excuser leur ton violent et leurs injures ${ }^{133}$. Mais dans tout cela le club ne paraissait point. "Ayant fait le résumé des différents avis contenus dans les lettres arrivées de Saint-Domingue, le président a dit que dans ce premier moment d'effroi, de consternation, ou du moins d'inquiétude qu'inspirent des nouvelles si alarmantes, il était impossible de prendre aucune détermination"134. Le club donc se tenait coi.Il recueillait les nouvelles, les gardait pour sa publicité, s'employait en entrevues, en échanges de renseignements.

La réponse de Moreau de Saint-Méry n'eut pas tout le succès attendu sur la décision du Comité de vérification. La conclusion de Grégoire fut adoptée dans son principe. Les gens de couleur étaient autorisés à se choisir des délégués. Seulement on ne leur en accorderait que deux au lieu des cinq demandés par Grégoire. Mais le droit de députer à l'Assemblée leur était formellement reconnu. C'était le principal.

Cette décision fit pousser de nouvelles clameurs. Le vote du Comité était un succès pour Grégoire. Cocherel crut nécesssaire de reprendre la réponse de Moreau de Saint-Méry. La colère s'oublie en répétitions. Les Observations de M. de Cocherel député de SaintDomingue sur la demande des mulatres ${ }^{135}$ étaient d'abord une protestation contre la décision du Comité de vérification et contre l'illégalité de la mission que se donnaient les délégués des sangmêlés de Paris. Cocherel conteste la régularité de leurs procurations: "Quels sont ces gens? Sont-ils colons? Les procurations qui énoncent

131. Brette, 345 .

132. Dont il y eut deux éditions, une parue chez Belin (décembre 89) de 55 pages in-8, et l'autre, (s.l. 26 décembre) de $68 \mathrm{p}$. in-8.

133. J. Raimond, Reponse aux Considérations de M. Moreau de Saint-Méry... sur les colonies (Paris, 12 mai 1791), 68 pages, in-8.

134. AN, W 15. Procès-verbal du 22 décembre 1789.

135. (Paris, s.d.), in-8, 18 pages. 
leurs pouvoirs ont-elles été passées devant notaires?"' Les vieux arguments revenaient. Puis un tourbillon d'articles s'accumule audessus de la question des colonies, après une nouvelle motion de Grégoire pour défendre à la Constituante, le 30 janvier, la conclusion de son Mémoire. Mosneron de Launay, député extraordinaire du commerce de Nantes et l'abbé Fauchet haussent bientôt le débat. Mosneron trouve à placer une défense de l'esclavage dans un supplément du Journal de Paris (24 janvier). A l'abbé Fauchet les Révolutions de Paris ouvrent leur tribune qui porte loin ${ }^{136}$ Mais tout cela n'émeut guère le club. Il sait que la motion que devaient faire les Amis des Noirs pour l'abolition de la traite n'aura pas lieu pour le moment (16 janvier). Il sait bien surtout qui a travaillé à obtenir ce silence ${ }^{137}$.

Il laisse parler. Les mulâtres ont obtenu deux députés. Les libres vont être admis à l'exercice des droits politiques, et bientôt assimilés aux blancs. C'est ce que dit le Comité de vérification. Mais c'est parole morte car la décision du Comité ne sera jamais notifiée à l'Assemblée Constituante. Le rapporteur, Grellet de Beauregard essaya bien de présenter son rapport Les députés de Saint-Domingue firent chorus avec les colons pour l'en empêcher. Il y eut pression du dehors. Il revint deux fois à la charge en séance. Deux fois les clameurs des Américains couvrirent sa voix. "Les colons et leurs partisans, dit Raimond, firent un tel tapage qu'il lui fut impossible de se faire entendre; alors il nous déclara qu'il renonçait à faire ce rapport"' ${ }^{138}$. Au club on pouvait se frotter les mains.

$$
\text { *** }
$$

Dejoly tourna ses batteries. Il s'adressa à la commune de Paris.

En janvier l'hôtel de ville avait déjà entendu des discussions sur le problème de l'esclavage ${ }^{139}$. La venue du président de la Société des colons Américains, le soir du 1er février, n'avait rien d'insolite

136. No 29 du 23-30 janvier, p. 41.

137. AN, Dxxv 85/826.

138. J. Raimond, Veritable origine des troubles de Saint-Domingue (Paris, 1797), 19 et Lettre des citoyens de couleur d MM. les membres du Comité de vérification (Paris, Lottin, s.d.), 7.

139. Supplique des citoyens de couleur... tendante à obtenir un jugement (30 janvier 1790) (Paris, s.d.) in-8, 12 pages. 
"à la tête d'une députation nombreuse de citoyens de couleur", une trentaine de libres, dont la moitié était des noirs ${ }^{140}$. Dejoly était lieutenant de maire. Ils venaient lire une adresse imprimée, prier la Commune d'employer sa médiation et celle de ses députés à la Constituante pour obtenir une décision au sujet de l'admission des libres aux droits politiques des blancs ${ }^{14}$. Cette démarche commune des mulâtres et des noirs libres répliquait à la manœuvre de Cocherel et du club, montrait que tous les libres étaient unis.

Brissot était là comme membre de la Commune et porte-parole des Amis des Noirs. Il monta à la tribune et supplia qu'on s'occupât de l'affaire sur le champ. L'Assemblée, le public étaient dans l'enthousiasme. Mais "la sagesse prévalut". La question fut ajournée au 6 février. Le débat du 6 fut ajourné au 11 ${ }^{142}$. On avait eu le temps de se préparer des deux côtés. Le 7 , Dejoly envoie un exemplaire de sa dernière adresse et une invitation à tous ses collègues de la Commune. Il leur recommande la cause qu'il défend, "que l'on veut confondre avec celle des esclaves, quoique ces deux demandes n'aient rien de commun"143. Le soir du 11, tous ses amis étaient à l'hôtel de ville. "L'intérêt de cette séance se trouvait accru par la présence des citoyens de couleur qui composaient la députation, par l'affectation louable que chacun marquait à les traiter en frères et à les faire jouir d'avance des droits qu'on leur refuse avec tant d'opiniâtreté dans leur pays et qu'ils sollicitent de l'Assemblée Nationale ${ }^{144}$. La lutte serait vive.

Devant ce public bruyant de sympathie "Dejoly expose les différents motifs qui déterminent la Commune à soutenir les libres. Puis Brissot lit un long discours. Il étend immédiatement la question, y joint celle de la traite, soutient que les planteurs sont des descendants de brigands, les accuse de chercher à exciter les ports et les places de commerce contre les gens de couleur. Ferrand de Baudière

140. Adresse des citoyens de couleur... à l'Assemblée générale des représentants de la Commune de Paris le ler fêrier 1790 par M. de Joly, (s.l.n.d.) in-8, 15 p.

141. S. Lacroix, Actes de la Commune de Paris pendant la Revolution - 1ère série - (8 vol., Paris, 1896), III: 662.

142. Ibid., IV: 63.

143. Lettre de Joly... 7 février 1790 (s.l.n.d.) in-8, 3 p. - AN, Dxxv 110, 876, pièce 11).

144. Moniteur, 16 février. 
est un martyr de l'humanité. L'on court à un soulèvement des mulâtres, à la formation d'un État séparé. Il rassure cependant l'assemblée en démontrant que la meilleure protection contre le danger de cette sécession que semblent préparer les colons, est l'émancipation de tous les mulâtres encore esclaves.

Marchais, auditeur des Comptes, représentant du district de Saint-Louis en l'île, releva la discussion interrompue par une autre question. Il n'était pas du club Massiac, mais il en résuma le point de vue. Évidemment la traite et l'esclavage étaient tout le contraire de la justice et de la morale. Mais ces maux nécessaires à la prospérité des colonies, il était impolitique de les détruire. L'or anglais, à coup sûr, entretenait "cette effervescence philosophique". L'Angleterre jalouse de nos îles, avait intérêt à un affranchissement général où elle perdrait moins que nous. L'argumentation n'avait rien de neuf. Les rôles avaient été répartis pour répondre à la première partie des assertions de Brissot.

La véritable bouche du club fut ce soir-là Poitevin de Maissemy, maître des requêtes, ancien directeur de la Librairie. Il était du club et avait, sans aucun doute, reçu mission de répliquer. Il prit la parole comme propriétaire de Saint-Domingue et son discours fut aussi rude que celui de Brissot. Il avança avec ordre, écartant l'un après l'autre les dires de Brissot, opposant des Adresses à celles qu'avait lues Brissot, qualifiant les Amis des Noirs de "novateurs incendiaires peut-être vendus à l'Angleterre". Mais surtout la question n'était pas de la compétence de la Commune. Il était inconcevable que Paris voulat "influer sur le régime de pays à 1,500 lieues de ses murs". En emmêlant la question de la traite avec celle des libres, la motion de Dejoly offrait le danger de diviser la France et d'exposer Paris à l'inimitié des villes de commerce. Au vrai, ce n'était pas Dejoly qui avait fait cette confusion.

Maissemy s'amusa à opposer le rôle réduit, volontairement modeste des colons réunis à Paris, à La Rochelle et à Bordeaux, à la conduite arrogante des mulâtres. Les colons que Dejoly présentait comme les ennemis des gens de couleur s'étaient bornés à supplier la Constituante de suspendre toutes décisions à l'égard des colonies jusqu'à ce qu'elle fat mieux éclairée par les assemblées coloniales. Les mulâtres s'étaient permis de présenter des cahiers, de proposer des lois - leurs lois - à l'assemblee de la Nation fran- 
çaise. Pourtant, d'un côté, il y avait six cents blancs ${ }^{145}$ les principaux propriétaires de l'île, de l'autre quelques-uns des gens de couleur qui résidaient en France, infime part de cette classe. En agissant ainsi pouvaient-ils oser dire qu'ils ne cherchaient pas à former une "corporation", une classe particulière, toute chose contraire aux récents décrets qui abolissent toute distinction d'ordre, tout corps. L'argument était cynique.

Il convenait d'adresser ces demandes aux assemblées des colonies. Si elles les accueillaient, comme il était vraisemblable, tout était terminé et régulièrement. Sinon, les gens de couleur pourraient en appeler à l'Assemblée Nationale. Ce que Maissemy n'ajoutait pas, c'était que les colons de France s'arrangeraient pour que le débat ne revînt pas devant la Constituante.

Toute spécieuse qu'elle était, cette opinion fit une vive impression sur la Commune. Plus de vingt voix demandèrent la question préalable. L'un proposa d'applaudir au zèle du défenseur des libres, mais sans délibérer sur leur demande. Un autre, d'ajourner indéfiniment la question. D'autres de renvoyer cette affaire devant les districts. Le plus favorable voulait inviter les députés de Paris à se souvenir du malheureux état des gens de couleur dont nulle loi ne prononçait l'exclusion.

La demande de la question préalable parut à Dejoly et à Brissot un outrage aux droits de l'humanité. Il était visible que la majorité se croyait "suffisamment éclairée". Dejoly consulta les commissaires des libres et se drapa avant de se retirer: "Il avait cru honorer l'assemblée de la commune en lui proposant une démarche glorieuse en faveur d'une classe d'hommes malheureux et opprimés, mais il ne voulait pas que ses registres fussent souillés par la mention d'une question préalable sur un objet qui intéressait si essentiellement la justice et l'humanite"'146.

Cette joute de trois heures se terminait par le succès des principes du club. Au reste, ce débat fini, le club parut rentrer dans sa coquille. Le 12 février, quand le Patriote Français s'emportait encore, il décida de ne "pas entamer de discussions littéraires" avec

145. Maissemy n'exagérait pas ce nombre.

146. Moniteur, 16 février. 
les Amis des Noirs et les journaux. Mais il inspirait les siens ${ }^{147}$. Les Révolutions de Paris qui avaient laissé passer la lettre de protestation de l'abbé Fauchet, se reprenaient sans transition" ${ }^{148}$. "On pense bien que la discussion des motions au moins inutiles de l'abbé Fauchet, a dû faire perdre beaucoup de temps aux commissaires nommés pour dresser un plan municipal... Le sieur de Joly... a présenté à l'assemblée... la demande... de l'admission de leurs députés à l'Assemblée Nationale". Loustalot détestait Brissot et n'aimait guère "le Joly". Son persiflage était l'interprète de la haine des colons. En voici le ton:

Cette démarche était concertée avec le sieur Brissot se disant de Warville, mandataire provisoire de la ville, frère terrible du Comité de recherches, président de la Société des Amis des Noirs, et de plus, journaliste. Cet honorable membre a, comme on voit, embrassé plusieurs moyens à la fois pour parvenir à la célébrité et à la fortune. Il préconise comme journaliste tout ce qu'il fait ou fait faire comme président des Amis des Noirs ${ }^{19}$. Comme chef des frères inquisiteurs il épouvante quiconque serait tenté de lui prouver qu'il n'est comme journaliste qu'un pédant bouffi de ses petits ouvrages et de ses petits voyages, recompilant aujourd'hui ce qu'il avait compilé avant la Révolution pour faire croire qu'il était grand politique, rouant impitoyablement quiconque ose dire que le comité des recherches est une institution exécrable et ceux qui l'exercent des citoyens méprisables; imprimant, braillant que l'Assemblée Nationale se déshonorerait si elle n'adoptait pas ce que la Sociêté des Amis des Noirs a décrété, voulant être à tout prix un des coryphées des enragés, qui n'ont pas plus besoin de lui pour faire la Constitution, que les noirs pour devenir libres; le Brissot ne cessera pas de remuer, de mentionner, d'intrigailler, qu'il n'ait vu cinq ou six enfants du Congo assis dans une assemblée nationale de France. ${ }^{150}$

L'ironie fiacile de Loustalot faisait remarquer que si les noirs étaient capables d'entrer au Corps Législatif, ils n'avaient pas

147. AN, Dxxv 89, P.V. du 12 février.

148. No du 13-20 février, p. 21-23. Il y avait déjà des extraits narquois et aigresdoux de la presse anglaise dans le no 31 des 6-13 février.

149. Brissot n'en était que le secrétaire. Il s'agit du Comité des recherches de la Commune.

150. No 32 , p. $37-39$. 
besoin d'avocat, et qu'il ne convenait pas plus a la Commune de Paris qu'à celle de Montmartre d'appuyer la cause des gens de couleur auprès de la Constituante, et il terminait sur le thème maintenant. banal, de l'indispensable autonomie des colonies, voire de leur sécession. De même que son article d'octobre était un écho des vœux du club Massiac, celui de février était l'écho du discours de Maissemy: "Et c'est ainsi que ces MM. s'occupent du plan de municipalités". Cette violence offensive s'oppose à la douceur du ton avec lequel le même journal rend compte au même moment de l'affaire de Favras, frère de Mahy de Cormeré, colon et membre du club. ${ }^{151}$ Tout ce numéro est à la dévotion des colons qui y font insérer une longue lettre d'un de leurs amis, nommé La Sauzée, qui répétait que les esclaves étaient plus heureux que les paysans ou les journaliers d'Europe et que "réclamer la liberté des nègres en Amérique équivalait à demander aux Français le partage des terres et des fortunes et que l'émancipation aboutirait d'ailleurs à la perte des colonies".

Une délégation d'une trentaine de membres de l'armée patriotique de Bordeaux, venue protester auprès de la Constituante contre tous les projets de réforme coloniale apportait au club un secours fort attendu d'opinions sollicitées ${ }^{152}$. Les Bordelais étaient reçus le 18 fevrier par le maire et la Commune de Paris. Sous le couvert "d'une alliance des gardes nationales", celle de Bordeaux se levait contre l'affranchissement des noirs qui menaçait la vente des vins de Guyenne. La réponse de Bailly fut aimablement évasive. Tout le monde comprenait que cette délégation venue du bout de la France et qui ne parlait point directement des gens de couleur, était une contreattaque.

La question fut ajournée deux fois par la Commune. Mais elle rebondit devant les districts. Le 22 février, celui des Filles-SaintThomas, inspiré par son président Magol et par Milly, colon de la Martinique, adopta la thèse des Bordelais et du Commerce de

151. Les Réolutions de Paris parlent de cet événement sur un ton semblable à celui que prend sur le même fait la Chronique de Paris, de Rozoi, toute dévoúce au club.

152. S. Lacroix, IV; 143; le Patriote Francais, no 184, (8 février); M. Lhéritier, Liberté, Les Girondins. Bordeaux et la Revolution française (1789-1790). (Paris, la Renaissance du livre, 1947), 127. 
Rouen ${ }^{153}$. Le district des Carmes déchaussés, dont le président se trouvait être aussi un colon de l'hôtel Massiac, P.-A. Lohier ${ }^{154}$, fit de même le 26 et les Filles-Dieu, le lendemain. La pétition de Bordeaux fut aussi discutée au club des Jacobins. Mais on voit mal dans quel sens ils se prononcèrent ni même s'ils se prononcèrent. Mirabeau fit un beau discours en faveur des noirs. Mais ici les esclaves n'avaient rien de commun avec les libres. Les gardes nationaux bordelais furent admis à répondre ${ }^{155}$.

Le 25, la Constituante reçoit les Bordelais. Le terrain, les circonstances parurent peu propices au club qui avait d'ailleurs, dès le début, trouvé la démarche de Bordeaux maladroite dans sa forme. Il s'en tenait à sa politique de silence à tout prix. Moreau de SaintMéry demande et obtient le renvoi de l'examen de l'adresse au ler mars. Un courrier arrivait juste des colonies. On le savait gros d'événements et de menaces ${ }^{156}$. Il était indispensable de le connaitre pour aviser à répondre aux Bordelais. Quoi de plus sage ? Moreau de Saint-Méry qui ne fréquentait plus l'hôtel Massiac depuis le mois d'octobre 1789 s'était montré l'homme du club.

\section{$\mathbf{X}$}

\section{AU COMITÉ DES COLONIES (mars 1790)}

Le ler mars, le Comité des Rapports venait à peine de recevoir du ministre communication des pièces relatives aux colonies. Le rapporteur n'était pas prêt. On attendit au lendemain. On savait que ce serait la grande partie.

Le 2 il fut évident que la question de la traite et des libres dominerait tout. Des amis du club, tel Begouen-Demeaux, du Havre, voulaient gagner encore du temps et repousser la discussion au lendemain du rapport du Comité d'Agriculture et du Commerce. Mais

153. S. Lacroix, IV: 359; Mogol, Discours sur la question relative à la liberté des nègres prononcé en assemblé générale du district des Filles Saint-Thomas (s.l. 1790), in-8; de Milly, Discours prononcé le 20 férier 1790... (Paris, 1790), in-8, 38+6 pages.

154. Fils d'un conseiller au Conseil du Cap. AN, Colonies E. 228.

155. Lheritier, 136.

156. Archives parlementaires, IX, 700. 
Camus, appuyé par Grégaire et Goupilleau fit adopter rapidement, malgré Maury, interprète du club, la création de ce Comité des Colonies demandé en vain trois mois auparavant. Il préparerait des rapports sur toutes les graves questions. L'opposition des colons n'avait pas été unanime. Si tous étaient d'accord pour voir dans un comité un bon moyen de "retenir" les questions, de les étudier à loisir, il en restait qui considéraient cette création comme "dangereuse dans les circonstances". D'ailleurs combien de colons, même à Paris, demeuraient hostiles à l'idée d'un club!

Mais la composition du comité choisi le 4 mars était pour rassurer le club. Sur les douze députés de ce Comité il y avait quatre colons: Payen de Boisneuf, J.-B. Gérard, le comte de Reynaud, et Pellerin de la Buxière; deux négociants coloniaux, en même temps propriétaires de Saint-Domingue: Garesché, de Saintes, et BegouenDemeaux. Alexandre de Lameth, dont le frère aîné possédait du chef de sa femme une grande sucrerie dans le sud, et Barnave étaient au courant des choses de Saint-Domingue. Nompère de Champigny - le futur duc de Cadore - officier de marine, avait visité la colonie; Alquier, négociant rochelais n'était pas étranger aux affaires coloniales. Seuls Le Chapelier et Thouret étaient sans lien avec le monde des planteurs. Le Comité choisissait Barnave pour rapporteur. Son rapport devait être prêt pour le 8 . Il y fallait du zèle, de la facilité, des connaissances. Barnave promettait tout. Il ne devait pas être cet ignorant en choses coloniales qu'on a dit. Un parent de sa mère comptait à Saint-Domingue parmi les plus grands colons. C'était Bacon de la Chevalerie, qui d'ailleurs faisait beaucoup parler de lui depuis le début de la Révolution. Autour de Barnave adolescent, bien des conversations avaient roule sur Saint-Domingue, ses esclaves, ses héritières et sa richesse. Nul besoin de faire intervenir ici le lien qui unit Barnave aux Lamethe en $1789^{157}$. Barnave a dô entrer directement en relations avec le club, où un seul des Lameth s'était montré le 20 août 1789 et dont un seul sera membre, mais plus tard, en novembre 1791. C'est Billard, le président du club qui fut en rapport avec Barnave et communiqua avec lui, au dire de L. Deschamps par la voie de Lafon de

157. L. Leclerc, "Les Lameth et le club Massiac", Ann. hist. de la Rév. française (sept.-octobre 1933), 461-463. 
Ladébat, négociant bordelais, propriétaire colonial et député ${ }^{158}$. Du club à Barnave on avait préparé les instructions de la Constituante poutr la convocation des Assemblées coloniales, et en somme la Constitution coloniale. Jusqu'à quel point étaient-ils tombés d'accord sur l'admission de certaines catégories de libres à l'égalité avec les blancs? Nous n'en saurons sans doute jamais rien. L'entente ne devait pas être parfaite sur tous les points, mais on se connaissait bien. On se rendait service. Ce ne fut pas hasard si Barnave et un Lameth se trouvèrent membres du Comité. Le club instruisit, renseigna Barnave. Mais en somme rien ne permet de dire quelles étaient les idées de Barnave sur la question des libres avant mars $\mathbf{1 7 9 0 .}$

Barnave aussi prépara son rapport après plusieurs conférences avec les représentants de la garde nationale de Bordeaux. Les Bordelais avaient leur projet de décret tout prêt. Selon leur dire, le projet que Barnave vint lire aux Jacobins dans la séance extraordinaire du 7 mars était, à une phrase près, conforme au leur. Ils en demandèrent le retrait ${ }^{159}$. Pendant tous ces jours Barnave fut aux écoutes de tous les intérêts et de toutes les craintes des colons, mais aussi des marchands et des ports. Le point de vue de Bordeaux était plus chaudement défendu que tous les autres par la présence active des délégués de sa garde nationale. Et l'on sait que l'opinion bordelaise n'était pas farouchement hostile aux libres. Pourtant le projet de Barnave chercha à satisfaire tout le mơnde, à calmer la peur des planteurs, à satisfaire les ports et les libres. Seulement il comptait sur deux incertitudes, sur les divisions des libres à la colonie qui empêcheraient l'accès de tous à l'égalité, et sur le jeu du temps qui dénoue les passions et les crises. Dans ce rapport, rien n'était décidé sur le brâlant problème de la traite et de l'esclavage. Il n'en était pas question, et tous ces silences étaient réfléchis; ils n'étaient muets pour personne. La Constituante s'excusait de tous les essais de lois qu'elle avait tentés jusqu'alors pour les colonies. Elle n'avait entendu ne rien innover dans le commerce soit direct ou indirect de la France avec les îles. Et ce petit mot rassurait ceux

158. Les colonies pendant la Révolution (Paris, 1898), 88. Nous n'avons pas trouvé trace de l'action de Lafon Ladébat dans les papiers du club, ni de celle des Lameth.

159. Arch. Mun. Bordeaux, Inventaire, t. IV: 219, H 43, lettre des délégués de la garde nationale du 4 mars et rapport du 18. - Lhéritier, 138. 
qui savaient entendre, car il était du langage du club et de ses directeurs quand ils parlaient de l'esclavage et de la traite.

Bien sûr, promettre aux colonies des lois adaptées à leurs convenances locales et particulières, c'était rendre incertaine la condition des libres, plutôt c'était la subordonner au bon vouloir, là-bas, des colons. Et Barnave ne pouvait pas ignorer quels étaient les vouloirs des colons. Mais on pouvait espérer alors un peu d'assouplissement de leur résistance, et Barnave savait que les négociants étaient plus opportunistes et qu'ils avaient souvent eu le dernier mot dans les différends coloniaux. Les colonies feraient connaître leurs vœux, c'est-à-dire leurs volontés, à la Constituante par la voie d'Assemblées coloniales "librement élues par les citoyens". Librement élues était clair, et rappelait que les élections devaient être publiques et régulières pour être valables, et non comme celles de 1789 pour les députés de la colonie aux États généraux. Mais quels étaient les citoyens? Les gens de couleur étaient-ils citoyens? Le mot s'ouvrait à toutes les espérances. Aussi à toutes les passions.

Pétion, des Amis des Noirs, et Mirabeau, qui avait à répéter devant un large public son discours des Jacobins sur l'esclavage, essayèrent d'obtenir une définition. En vain. Le mot ne fut pas défini, car on craignait une discussion générale du fond du problème. Barnave n'en voulut pas car les débats avaient dû être déjà très vifs aux séances du Comité. Quelles étaient les intentions de Mirabeau? C'était assez dans ses procédés de noyer son opinion sur une question pratique dans des développements sur des principes généraux.

Barnave - et le club derrière lui - emporta le vote de son projet le 8 mars. Le décret accordait indirectement aux colons une constitution particulière. Barnave comptait satisfaire les autonomistes. Le décret mettait fin "au régime ministériel". La concession était grande. La Constituante espérait qu'en retour les colons laisseraient au mot citoyens, un généreux flottement qui permettrait l'immédiate entrée des quarterons propriétaires aux assemblées primaires, et aux autres libres une amélioration de leur sort. Barnave et l'assemblée faisaient confiance au temps et à l'espace. Ils coyaient se débarrasser de la question en la renvoyant d'où elle leur était venue. En fait ils s'en remettaient à la force, aux passions. Leur décret serait ce qu'on l'interpréterait.

Il allait être immédiatement gauchi à Paris même. Il disait 
"quiconque travaillerait à exciter des soulèvements contre les colons serait déclaré criminel envers la nation". Les Amis des Noirs désormais n'avaient qu'à demeurer cois. ${ }^{160}$ C'était bien les colons, les colons français, non les américains, que cet article protégeait. Et le jour même où le décret était adopté le district des Filles Saint-Thomas, dont Brissot était membre, répondit à un mémoire défensif du secrétaire des Amis des Noirs par l'offre d'une généreuse amnistie. Mais l'initiative était de Milly et masquait une menace ${ }^{161}$. Le club se faisait bon prince et souriait à l'avenir. Il resta silencieux, ne se déclara pas franchement satisfait, pour mieux cacher ses connivences avec les hommes du décret. Les Amis des Noirs, eux aussi, modérant leur propagande, rentrent dans une obscurité de quelques semaines ${ }^{162}$.

Cependant une instruction devait suivre qui inspirât l'application du décret. C'est la fameuse instruction du 28 mars. Parce qu'elle sortit d'un nouveau rapport de Barnave on attribue à Barnave tout le mal qu'elle fit. Une nouvelle imprécision venait s'ajouter à celle qui recouvrait le mot citoyen du premier texte. Mais l'histoire de la rédaction de cette instruction n'est pas faite, qui sera difficile et nuancée. A peu près certainement elle diminuera la part de Barnave dans l'intention ambiguë de l'article 4. Beaucoup de choses nous échapperont toujours.

Une intervention très certaine, que nous ne connaissons que par une lettre de Gérard au Comité des Cayes ${ }^{163}$ est un nouveau mémoire que les gens de couleur déposèrent le 14 mars au bureau du Comité par l'entremise de Dejoly. Ce dépôt avait été précédé d'un plaidoyer qui fit une forte impression sur Thouret président du comite "ainsi que sur la plus grande partie des autres membres ${ }^{164 " . ~ D ' a p r e ̀ s ~ c e t t e ~}$

160. Archives parlementaires, XII: 627.

161. S. Lacroix, IV: 379.

162. Adresse du 10 avril 1790. AN, Dxxv 89/842 bis.

163. Du 15 mars. Bl. Maurel, "Un député de Saint-Domingue à la Constituante J.-B. Gérard", Revue d'histoire moderne (1934), 247.

164. Sans doute est-ce les Réclamations des citoyens de couleur... sur le décret du 8 mars [ du 10 mars ] (s.l. nill.d.), in-8, 23 p. 
lettre la majorité du comité aurait été pour l'accord de l'égalité aux libres. Ce furent Payen de Boisneuf, Pellerin de la Buxière, Reynaud, Garesché et Gérard lui-même, qui demandèrent l'atténuation du mot citoyens, et son remplacement dans l'instruction par celui de personnes. Ce qui prouve que le mot citoyens était de sens très net dans l'esprit du comité et que Barnave était parmi ceux qui voulaient le préciser entre le 8 et le 28 mars. C'est "pour ne pas éveiller le mécontentement des colons dans un moment où tant d'autres inquiétudes les tourmentent "et pour" leur laisser le mérite de la faculté d'exercer un acte de générosité très propre à inspirer aux gens de couleur des sentiments d'affection et de reconnaissance" qu'on laissa les choses dans une vague que Barnave ne paraît pas avoir demandé et qu'il n'était pas seul, tant s'en faut, à souhaiter. La formule faisait appel à l'esprit politique des colons à l'égard des petits blancs en même temps qu'à l'égard des gens de couleur ${ }^{165}$.

$\mathrm{Au}$ vote de cette instruction Grégoire demanda les précisions que le Comité avait redoutées. Les libres jouiraient-ils du droit de représentation? Mais Cocherel et les colons avaient parfaitement entendu la pensée du rapporteur, et Cocherel déclara immédiatement que le vœu des députés de Saint-Domingue était opposé à ce droit. Sur quoi Charles de Lameth et Gérard réclamèrent la fermeture de la discussion "sur la proposition indiscrète de $M$. l'abbé Grégoire" et la clôture fut votée. L'Assemblée se confiait à l'avenir et aux blancs. C'était le plus facile. Les mulâtres après sept mois d'attente se retrouvaient en face de leurs adversaires, chez eux, en champs clos. La Constituante s'en lavait les mains. Ce n'est pas du sang qui lave les mains.

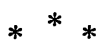

Beaucoup donc s'y trompèrent, les gens de couleur les premiers, au moins au début, car ils avaient obtenu au moins le principe, à quoi s'accrocher, sur quoi s'appuyer pour refuser d'obéir et de se battre. Ils respirèrent profondément ce qu'ils croyaient un air nouveau, mais sans se griser.

165. Bl. Maurel, op.cit., 247. 
Le 29 mars Dejoly faisait part de son triomphe à la Commune ${ }^{166}$ :

Messieurs, lorsque j'eus l'honneur de vous présenter les citoyens de couleur et de réclamer en leur nom votre appui auprès de vos députés à l'Assemblée Nationale, je contractai l'engagement de vous faire participer aux succès qu'ils pourraient obtenir. Permettez-moi d'acquitter aujourd'hui ma parole.

La cause des citoyens fut jugée hier. L'Assemblée Nationale a rejeté par la question préalable la motion de $\mathrm{M}$. Cocherel, tendante à exclure nommément les citoyens de couleur des assemblées primaires.

Elle a ensuite décrété que toutes les personnes âgées de vingt-cinq ans accomplis propriétaires d'immeubles, ou, à défaut, domiciliées dans la paroisse depuis deux ans et payant une contribution, se réuniront pour former l'assemblée paroissiale.

Ainsi, messieurs, plus de distinction entre un homme libre et un homme libre, entre un citoyen et un citoyen. La nature reprend partout ses droits imprescriptibles.

Dejoly se méprenait un peu facilement, mais dans l'ignorance où nous sommes de son plaidoyer du 14 , nous devons le croire tout à fait sincère le 29 mars. Il était vrai que la motion de Cocherel avait été repoussée, mais il aurait été complet d'ajouter que celle de l'abbé Grégoire, qui voulait assurer l'éligibilité et le droit de représentation aux libres propriétaires, avait été repoussée par la même question préalable.

L'acharnement du club à empêcher le départ de Vincent Ogé et de Fleury, les achats d'armes d'Ogé en avril, montrèrent immédiatement qu'en France colons et gens de couleur entendaient l'instruction d'une manière contradictoire ${ }^{167}$. Quelques libres ne se faisaient pas d'illusions mais le plus grand nombre restait à l'espoir. C'était à Saint-Domingue que tout allait se décider.

Une assemblée coloniale se réunit à Saint-Marc, en avril, avant l'arrivée des décret et instruction. Les libres n'avaient pas pris part aux élections, ce qui n'avait encore rien de décisif. Mais en juillet, par application de l'instruction de mars, on dut confirmer cette

166. S. Lacroix, op. cit., IV: 520.

167. Procès-verbal du Comité du 11 mars au soir. AN, Dxxv 89/842 bis. 
assemblée et refaire des élections. Les libres y voteraient-ils ou non? Ils revendiquèrent leur droit. Le gouverneur Peynier, interrogé sur l'article IV, trancha en permettant aux marguilliers, en raison de l'usage, de ne pas recevoir les libres aux assemblées de paroisse. C'était opposer la coutume au texte de la nouvelle loi, et reconnaitre aux libres le droit d'assister à ces assemblées ${ }^{168}$. Les gens de couleur furent reconnaissants de cette réponse qui les rapprocha des agents de l'autorité métropolitaine. Mais ils ne votèrent pas. Toute l'Assemblee de Saint-Marc était là pour le leur interdire, et avec elle tous les comités de paroisse. Quand on apprit ce refus en France, il n'y eut plus à douter. Tout était à reprendre. L'optimisme de mars était loin. Dejoly ne pouvait s'y tromper. Pourquoi ferma-t-il les yeux, s'estima-t-il satisfait encore ? Le mystère ne doit pas être bien profond. Il $y$ avait intérêt. Il avait dit sincèrement en mars, la partie gagnée. Il le croyait. Il va continuer de le dire, mais sans le croire, pour se tenir à l'écart d'une cause devenue inextricable et qui soulevait la moitié de l'Amérique. "M. de Joly a abandonné notre cause, écrit J. Raimond à ses compatriotes de Saint-Domingue, en mars 1791, et il n'a plus rien fait pour elle." Et Raimond met en contraste le désintéressement et la constance de Grégoire, de Pétion, de Clavière et de Brissot ${ }^{169}$.

Devant ces reproches qu'on sut rendre publics, Dejoly tenta d'expliquer son attitude dans une longue lettre à Brissot, le 29 mars 1791, qu'il fit imprimer ${ }^{170}$. C'est une apologie de juriste. Il démontre que le décret et l'instruction ont été parfaitement rédigés et dans l'absolu il a raison. Mais les mots n'ont de sens que celui qui les unit aux circonstances. Clair en soi, le mot citoyens prêtait à quelque confusion en mars 1790. "On ne pouvait cependant, dit-il, joindre le mot libres à celui de citoyens, parce que d'une part ils se supposent réciproquement et que le mot libres supposerait nécessairement une classe d'hommes vivant dans l'esclavage". Un avocat est d'abord un homme qui sait se donner des raisons. Cela peut faire sourire,

168. Cabon, III: 40.

169. Brette, 334 et 403.

170. AN, Dxxv 110/876, Lettre de M. Dejoly, avocat aux Conseils, représentant de la Commune de Paris et defenseur des citoyens de couleur relativement aux decrets sur les colonies, d̀ l'auteur du Patriote francais (Paris, 29 mars 1791), in-8, 3 p. 
mais certainement la Constituante avait eu ici la pudeur des mots. Le mot citoyens avait été écarté "par crainte de reconnaître d'une manière indirecte l'ancienne distinction de classe". On avait craint qu'en admettant très ouvertement les libres aux colonies, on ne dat bientôt admettre aussi en France tous les citoyens passifs. Le discours de Maissemy le 11 février avait son écho. Dejoly estime que l'expression toutes les personnes n'offre aucune équivoque. Elle a voulu comprendre indistinctement tous les propriétaires: ce ne sont pas les mulâtres riches qui sont rejetés, mais tous les petits blancs. L'on n'avait pu nommer, évidemment, ni les uns ni les autres. La lettre de Gérard du 15 mars et les débats autour des conditions de vote aux assemblées coloniales lors de la préparation du plan de convocation au club Massiac, nous apprennent que ce n'était pas là une explication de fantaisie. Le flou de l'article IV s'éclaire en partie par la peur des petits blancs sans propriété.

Un an après le vote de la Constituante Dejoly se cherchait des arguments, s'attachait à la seule lettre du décret, se donnait raison. Il finissait par une parole d'espoir, comme le Comité des Colonies, comme Barnave. Mais ce mot prouvait surtout que l'affaire n'intéressait plus Dejoly. "Attendons tout du temps; espérons que bientôt il ne se trouvera personne, même dans les colonies, qui méconnaisse les droits de l'homme libre, qui prétende établir et encore moins perpétuer la plus légère différence entre le citoyen et le citoyen."

Une interprétation aussi optimiste d'un texte que contredisaient les faits et que tant de colons voulaient lettre morte, nous mène à une autre explication de l'attitude de Dejoly. Faut-il supposer qu'il ait été touché par l'action du club Massiac qui avait ses moyens particuliers de convaincre ses adversaires? Fut-il gagné et à partir de quel moment? Dejoly fait un demi-aveu dans sa lettre du 29 mars 1791. “On m'a fait entrevoir, et les membres du Comité colonial qui paraissaient le mieux disposés en notre faveur ont particulièrement insisté pour me faire sentir le danger de déclarer que les libres jouissaient des droits attachés à la qualité de citoyen actif". Ne serait-ce pas désigner Gérard, qui voulait qu'on s'attachât les libres ou même Barnave qui d'abord ne leur était pas favorable. Mais à côté de l'intervention du Comité des Colonies il y a eu l'intervention de cet on. Une pression s'est exercée sur Dejoly, dont on voit la direction et le poids, dès la fin de mars 1790 , peut-être dès la mi-mars. Il 
n'avait pas répondu jusqu'au bout à l'attente de Raimond et des libres. Mais Raimond avait-il eu à choisir entre un grand nombre d'avocats? Les nouveaux et les faibles ne sont pas difficiles en espérance.

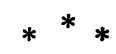

Là finissait comme le second épisode de la lutte pour l'égalité. Le premier avait été celui où jusqu'en août 1789 Raimond s'était lancé seul, sans réussir. La réunion des États généraux avait ouvert une autre forme de lutte. Raimond avait d'abord cherché et cru trouver dans la personne du comte de Jarnac un haut protecteur pour la cause qu'il défendait. Mais le protecteur s'était effacé sans qu'on eât rien obtenu, sans qu'il eât même probablement rien sollicité. Après septembre 1789 Raimond avait pensé qu'en mettant au premier plan un avocat de métier, aux fructueuses relations, un blanc, administrateur de Paris, il obtiendrait enfin les droits civiques pour les libres. Raimond et Dejoly, avec l'aide des Amis des Noirs, n'avaient pas complètement échoué, tant s'en faut. De la bataille de mars on rapportait un trophée, un droit, une manière d'engagement qu'on ne pourrait retirer que très difficilement.

La lutte va continuer, mais avec un autre tour et une double face. Jusque là l'action n'avait été menée qu'en France, avec l'appui lointain, discontinu, des libres de Saint-Domingue. Désormais forts du décret et surtout de l'instruction du 28 mars, de son article IV, ils vont réclamer violemment, prendre les armes, se trouver des appuis, officiels ou non. Des échauffourées incessantes tiendront l'attention en alerte. Des secousses partiront de Saint-Domingue. Raimond, d'autre part, sera auprès de l'Assemblée Constituante pour en recueillir les échos, les présenter comme preuve et protestation. Il agira au grand jour, comme un amplificateur, encore doux longtemps. Ce sera la troisième phase, celle du décret du 15 mai 1791, du contre-décret du 24 septembre 1791, enfin de la "loi du 4 avril."

Nous nous arrêtons ici au début de ce nouvel épisode, où disparaît Dejoly.

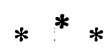


Tous ces mouvements paraîtront contés avec minutie. Il est vrài. Il le fallait pour être clairr et garder le fil dans cet écheveau de pétitions, d'intrigues et de polémiques. Mais on n'aura pas eu besoin de s'appliquer pour faire voir combien cette histoire est complexe et nous échappe par ses côtés. Ce n'est qu'un essai. Nous avons poussé une porte. Nous la laissons entrebaillée.

A plus tard. Ou à d'autres.

G. DeBIEN,
Le Caire, Egypte

N.B. Tous les abonnés à la Revue et leurs amis sont cordialement invités à la réunion générale de l'Institut qui aura lieu, le 14 avril procbain, à la Bibliotbèque Municipale de Montréal, 1210 est, rue Sberbrooke. On est également invité au banquet, le soir du 14 avril, à l'Hôtel. Windsor (Montréal). Prix du billet : \$3.00. Lire la Chronique à ce sujet, p. 605-606. 